\title{
Background Intensity Problems in High Resolution Defect Imaging
}

\author{
A. Howie, ${ }^{*}$ N. Jiang, ${ }^{* *}$ J.C.H. Spence**and J. Wu** \\ *Cavendish Laboratory, University of Cambridge, Madingley Road, Cambridge CB3 0HE, UK. \\ **Department of Physics and Astronomy, Arizona State University, Tempe, AZ 85287.
}

Most high resolution images of crystal defects contain appreciable background contributions from inelastic scattering, thermal diffuse scattering (TDS) and from elastic scattering in overlapping crystal regions or in disordered surface layers. Their significance, particularly for large-angle inelastic (Compton) scattering, was noted in the case of weak beam imaging before energy-filtering facilities were widely available [1,2]. The harmful effect of small-angle inelastic scattering on weak beam image contrast in thicker crystals was also demonstrated [3]. It still appears however that in most current weak beam work, energy filtering is not yet fully exploited for operating in thicker crystals and/or at still weaker beam conditions and thus potentially higher spatial resolution. From CBED experience, energy filtering could also be a significant step in defect image quantification.

Comparison in fig. 1 of bright-field and weak-beam EELS data shows for the latter the significantly larger contribution from Compton-scattered electrons above $50 \mathrm{eV}$. The difference (see inset) is reduced with smaller objective apertures but energy filtering is clearly also worthwhile. Diffraction space EELS (fig. 2) shows the angular dependence of both the zero loss and inelastic signals and their contributions to the background. In principle, though much more difficult, it would be possible also to remove the TDS component by holographic side-band weak beam imaging [4]. In the much easier strategy of simply subtracting the background intensity measured near the defect, it should be noted that there is a possibly significant correction, easily calculable by diffraction contrast theory, which should be applied because of the change in electron wave channeling at the defect.

In HAADF STEM imaging, TDS contributes the most important part of the signal but there is also a contribution from large-angle inelastic scattering which may be relatively more significant in lower $\mathrm{Z}$ materials [5-7]. Given the scattering angles involved, it is not entirely straightforward to measure this effect but there is in the meantime a simple recipe for computing the inelastic scattering differential cross section as a function of scattering angle [8]. Multiple scattering effects should also be included however. Such computations may be useful in clarifying the possible importance of energy-filtered HAADF imaging e.g. by using a small axial stop followed by post-specimen lens, aberration-corrected focusing of the ADF signal into the spectrometer.

\section{References}

[1] P.L. Gai and A. Howie, Phil. Mag. 31 (1975) 519.

[2] A. Howie, Proc. 39 $9^{\text {th }}$ EMSA Meeting, Claitor's Publishing, Baton Rouge (1981) 186.

[3] R.E. Schaublin and W.M. Stobbs, IOP Conf. Series 147 (1995) 203.

[4] C. B. Boothroyd and R.E. Dunin-Borkowski, Ultramicroscopy 98 (2004) 115.

[5] D.J. Eaglesham and S.D. Berger, Ultramicroscopy 53 (1994) 319.

[6] A.L. Bleloch, M.R. Castell, A. Howie and C.A. Walsh, Ultramicroscopy 54 (1994) 107.

[7] C B Boothroyd, R E Dunin-Borkowski and T Walther, Mater. Res. Soc. Proc. 466 (1996) 113.

[8] D.R. Penn, Phys. Rev. B35 (1987) 482. 


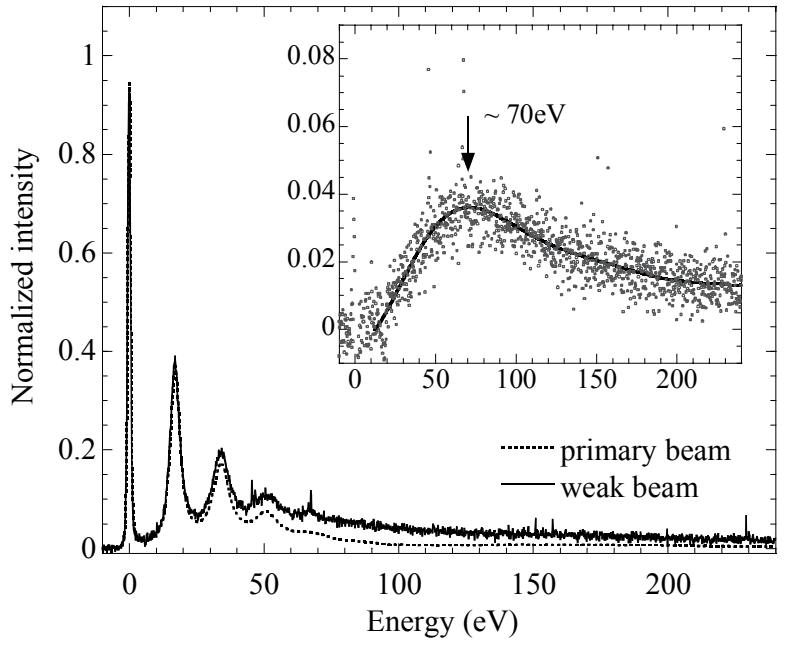

(a)

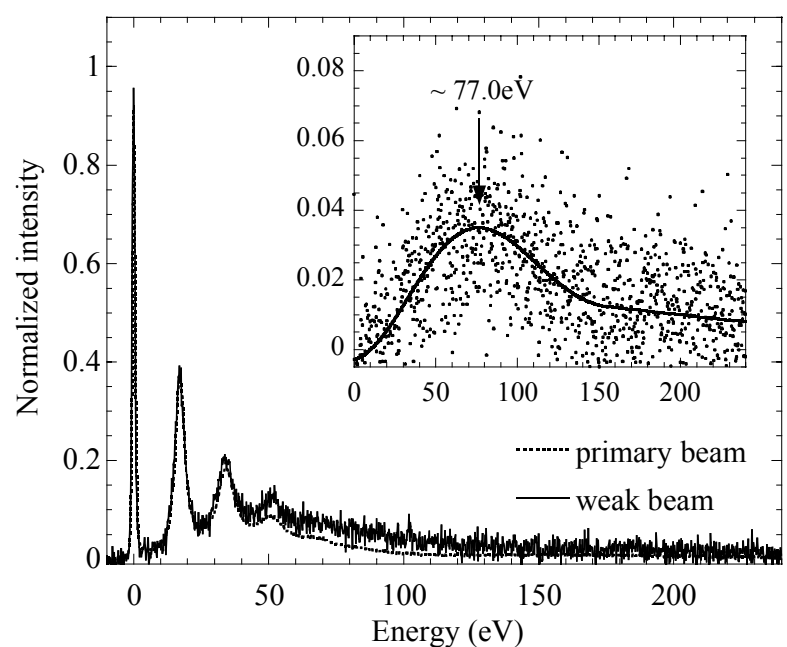

(b)

Fig. 1. EELS spectra recorded at $200 \mathrm{keV}$ in image mode with the $\mathrm{Si}$ crystal set to the $3 \mathrm{~g}$ Bragg condition while imaging $\mathrm{g}=(004)$. The primary beam (bright-field) and weak beam (dark field $\mathrm{g}$ ) spectra with a $7.6 \mathrm{mrad}$ collection semi-angle were normalized to the same zero loss intensity and their difference is shown in the inset. A smaller condenser aperture was used in (b).
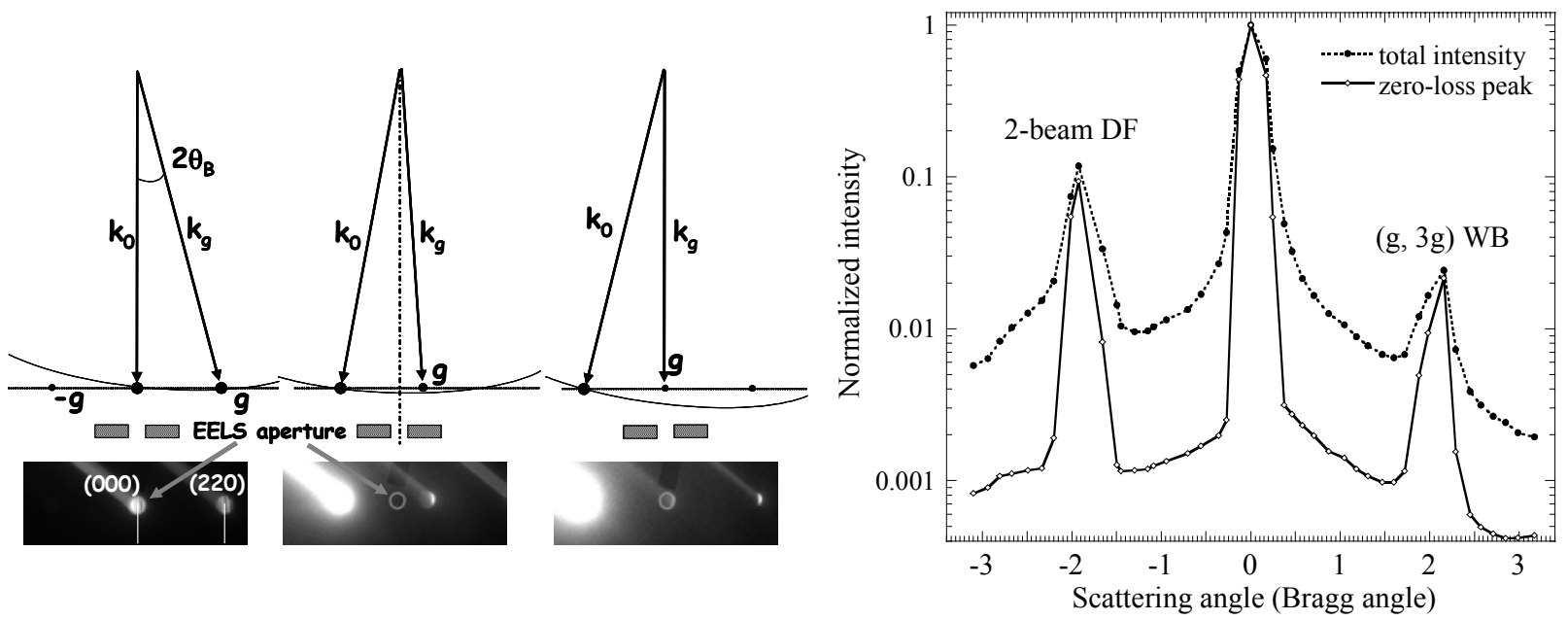

Fig. 2. Filtered and unfiltered signals vs. illumination tilt angle $\theta$ for an effective aperture semi-angle of $4 \mathrm{mrad}$. With axial illumination, the Si crystal was oriented to the $+\mathrm{g}$ Bragg condition. The peaks in the plot at $\theta / \theta_{\mathrm{B}}=-2$ and $\theta / \theta_{\mathrm{B}}=+2$ thus correspond to the $-\mathrm{g}$ strong beam Bragg beam and to the $(\mathrm{g}, 3 \mathrm{~g})$ weak beam respectively. For the 220 reflection $\theta_{\mathrm{B}}$ is $13.1 \mathrm{mrad}$ at $200 \mathrm{keV}$. Extrapolation under the Bragg peaks shows the substantial improvement in signal to background due to filtering although $10 \%$ of the background is from elastic scattering in surface layers and/or TDS. 